\title{
Therapie der Hepatitis C: Eine Erfolgsgeschichte
}

\author{
Die Herausforderung für den Arzt ist die Adhärenz des Patienten
}

Bis man sie erkennt, ist die Hepatitis C meist schon weit fortgeschritten, denn die Symptome machen sich erst spät bemerkbar. Die Sterblichkeit hat weltweit mittlerweile jene von HIV-Infektionen überholt. Hepatitis C ist die häufigste Ursache für chronische Leberentzündung, Leberzirrhose und Leberkrebs. Mit den neuen Porteaseinhibitoren nähert man sich nun der idealen Therapie an. „Die Therapie der Hepatitis C ist eine Erfolgsgeschichte“, stellte Univ.-Prof. Dr. Gabriele Moser, Univ.-Klinik für Innere Medizin III, Wien, anlässlich des Welt Hepatitis Tags am 28. Juli in Wien fest. „Eine sehr lange als chronisch geltende Krankheit ist jetzt heilbar.

Genaue Zahlen für die Inzidenz in Österreich liegen derzeit nicht vor. Man geht davon aus, dass etwa 500 symptomatisch Erkrankte pro Jahr diagnostiziert werden und etwa 2000 Erkrankte zwar diagnostiziert aber nicht behandelt werden, erklärte Univ.Prof. Dr. Peter Ferenci, Univ.-Klinik für Innere Medizin III, Klin. Abt. für Gastroenterologie und Hepatologie, Wien. Die Schätzungen für die Gesamtzahl reichen jedoch von 10.000 bis 150.000 . Als Risikogruppe gelten Personen, die vor 1992 eine Bluttransfusion erhielten, Personen mit i.v. Drogenmissbrauch - aktuell wie zurückliegend - und Migranten. In jedem Fall wird die

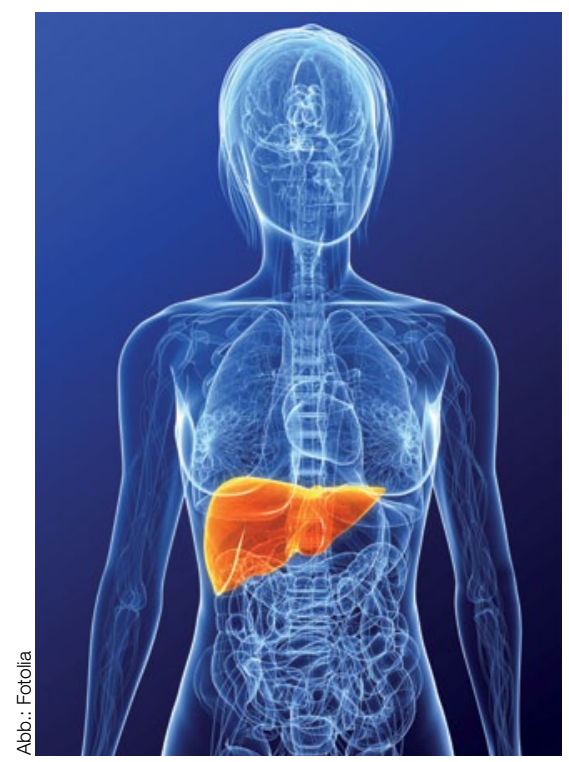

Ein Drittel der Patienten mit dekompensierter Leberzirrhose stirbt an Leberkrebs.
Infektion über Blut und Blutprodukte übertragen, während eine sexuelle Übertragung kaum möglich ist, stellte Ferenci klar: „Das ist der große Unterschied zur Hepatitis B.“

\section{Tückisch, weil lange ohne Symptome}

Eine akute Erkrankung verläuft in vielen Fällen völlig beschwerdefrei und heilt bei 20 bis 50 Prozent der Betroffenen von selbst aus. Bei 50 bis 80 Prozent wird sie jedoch chronisch - und es passiert, so Ferenci, „,in den erste 20 Jahren nach der Infektion nichts.“ Das Risiko, eine Leberzirrhose zu entwickeln, beträgt etwa ein Prozent pro Jahr. Ein Drittel der Patienten mit dekompensierter Leberzirrhose stirbt an Leberkrebs. Erst die Einführung der Interferone und schließlich die Kombination aus pegyliertem Interferon und dem Nukleosid Analogon Ribavirin brachte in der Therapie der chronischen Hepatitis $C$ einen ersten Durchbruch: Die Heilungsraten stiegen von 20 auf 70 bis 90 Prozent. Abhängig vom Genotyp. „Durch die Zulassung zweier neuer antiviraler Therapien im Jahr 2011 konnten“, so Ferenci, „die Erfolgsraten in Kombination mit dem bisherigen Therapiestandard weiter erhöht werden."

Insbesondere der Genotyp 1, der in Österreich am häufigsten vorkommt und bisher als schwierig zu therapieren galt, spricht auf die um den Proteaseinhibitor (Boceprevir oder Telaprevir) erweiterte Kombinationstherapie deutlich besser an. "Bei nicht vorbehandelten Patienten, die erstmalig eine Therapie bekommen, ist eine Verbesserung der Ausheilungschancen gegenüber der bisherigen Standardtherapie um etwa 25 bis 30 Prozent auf 70 Prozent erreichbar", erklärte Univ.-Prof. Dr. Harald Hofer, Univ.-Klinik für Innere Medizin III, Wien. Bei der großen Gruppe von bereits mit der klassischen Standardtherapie aus pegyliertem Interferon und Ribavirin vorbehandelten Patienten, die damit nicht ausgeheilt werden konnten, profitieren vor allem jene, die einen „Relapse“ auf die Vortherapie hatten. Die neue Dreierkombination kann hier eine Heilungsrate von bis zu 85 Prozent erreichen. Auch bei vielen „Null-Respondern“ lassen sich mit der Dreifachkombination Heilungsraten von 30 bis 40 Prozent erreichen, berichtete Hofer. Als Kriterien, die die
Heilungschancen beeinflussen, nannte Hofer das Vorliegen einer Leberzirrhose, bestimmte genetische Polymorphismen (z. B. IL-28B-Polymorphismus), Virusfaktoren wie die Höhe der Viruslast, der vorliegende Virussubtyp sowie das Ansprechen auf die Vortherapie. „Fortgeschrittene Fibrose bzw. Zirrhose und schlechtes Ansprechen sind“, so Hofer, „schlechte Prognosefaktoren. “ Faktoren, die die Progression der Krankheit erhöhen sind Alkoholkonsum, männliches Geschlecht, Übergewicht und höheres Alter.

\section{Die Lebensqualität ist schwer beeinträchtigt}

Sowohl die Diagnose als auch die Erkrankung und die Therapie selbst haben Einfluss auf die Lebensqualität, vor allem was die psychische Ebene betrifft. Die häufigsten Beschwerden aufgrund der Erkrankung selbst reichen in frühen Stadien von Gelenkund Kopfschmerzen, Gewichtsabnahme, Oberbauchbeschwerden bis zu Müdigkeit und Reizbarkeit. „In Spezialzentren weisen 60 Prozent der Betroffenen eine Depression auf, die meist innerhalb der ersten acht Wochen einer Interferontherapie auftreten", berichtete Moser. Typische Nebenwirkungen des Proteaseinhibtors sind Anämie und damit verbunden Müdigkeit, sowie Hautausschläge, Juckreiz und metallischer Geschmack im Mund. Eine sorgfältige Begleitung des Patienten ist daher für den Therapieerfolg entscheidend, ebenso wie eine möglichst frühe Therapie. Denn Patienten mit Depression weisen ein erhöhtes Risiko für einen Therapieabbruch von zehn bis 40 Prozent auf.

Nach wie vor gilt: Je weiter fortgeschritten die Erkrankung ist, desto unwahrscheinlicher die Heilung. Da die Nebenwirkungen in der Gruppe der schwer zu therapierenden Patienten hoch sind, ist in dieser Gruppe auch die Adhärenz am geringsten. „Das ist die Herausforderung für den Arzt", konstatierte Ferenci. Ziel ist: Die Interferonfreie Therapie mit Heilungsraten von 100 Prozent, wenig Nebenwirkungen und geringen Kosten. „Wir nähern uns“, erklärte Ferenci hoffnungsvoll.

Quelle: Pressekonferenz Welt-Hepatitis-Tag 28. Juli 2012. Hepatitis: Gefährliche Infektionskrankheit verliert ihren Schrecken, 25. Juli 2012, Wien. 